\title{
Performance on the PD test battery by relatives of patients with progressive supranuclear palsy
}

\author{
Kenneth B. Baker, PhD; and Erwin B. Montgomery, Jr., MD
}

\begin{abstract}
Article abstract-Objective: To determine whether there is a greater prevalence of asymptomatic first-degree relatives (FDR) of patients with progressive supranuclear palsy (PSP) performing abnormally on the PD test battery (PD Battery) compared to sex- and age-matched normal control (NC) individuals. The PD Battery incorporates tests of motor function, olfaction, and mood. It has high specificity and sensitivity in distinguishing mildly affected PD patients from NC individuals in previous studies. Methods: This test battery and regression analysis-derived scoring equations were applied to asymptomatic FDR. Results: Twenty-three FDR and 23 NC individuals were tested. Of the FDR, 39\% scored in the abnormal range, whereas none of the NC individuals achieved abnormal scores. This difference was significant. Further analysis demonstrated that the two groups differed significantly on a measure of simple reaction time. Conclusions: The proportion of FDR who demonstrated abnormal performance on the PD Battery was greater than NC individuals. Thus, the PD Battery may detect the asymptomatic carrier state or risk for PSP or a subclinical effect of a shared environmental exposure.
\end{abstract}

NEUROLOGY 2001;56:25-30

Previous work in our laboratory demonstrated that, relative to normal controls, asymptomatic firstdegree relatives (FDR) of individuals with PD show a higher rate of abnormal performance on a sensitive and specific battery of tests developed for the early detection of PD (the PD Battery). ${ }^{1}$ Given a putative role for genetics in the cause of typical $\mathrm{PD}$, that study raised questions regarding the possibility of detecting an asymptomatic carrier or sublinical state of the disease as well as the potential impact of such detection on future genetic and environmental research. The existence of similar issues and goals in progressive supranuclear palsy (PSP) led us to extend this work to the FDR of individuals with PSP.

PSP is a neurodegenerative disease with pathology principally in the basal ganglia and brainstem, although most levels of the CNS typically are involved to some degree. ${ }^{2}$ The cause of PSP is not known, and although there is some evidence for a familial subtype of the disease, it is still considered to occur sporadically. However, the term sporadic refers only to the lack of any pattern to its occurrence, and a genetic cause with low and variable penetrance may appear sporadic.

Interest in the possible role of genetics in PSP can

Additional material related to this article can be found on the Neurology Web site. Go to www.neurology.org and then scroll down the Table of Contents for the January 9 issue to find the title link for this article. be found in an increasing number of reports concerning cases of multiple family members with $\mathrm{PSP}^{3-7}$ as well as investigations into the possible role of the tau gene. ${ }^{8-17} \mathrm{~A}$ test battery capable of identifying the asymptomatic or subclinical carrier state of the putative gene(s) could facilitate work on identification of the putative genetic pathogenesis. Similarly, identification of subclinical states would facilitate identification of shared environmental exposures.

The PD Battery incorporates tests of motor function, olfaction, and mood. It has been shown to distinguish mildly affected and newly diagnosed PD patients from normal control (NC) individuals with high specificity and sensitivity. ${ }^{18}$ Preliminary prospective studies have shown that the PD Battery, given to individuals without sufficient symptoms or signs to make a diagnosis of $\mathrm{PD}$, is $89 \%$ specific and $71 \%$ sensitive in identifying those who will reach clinically diagnosable PD within 2 years of testing. ${ }^{19}$ In this study, we examined the performance of a group of FDR, primarily children, of individuals with PSP on the PD Battery. In addition, the test battery also was applied to age-matched $\mathrm{NC}$ individuals who did not have a family history of PSP or PD. The prevalence of abnormal performance on the PD Battery was compared between the two groups. Additional analyses were performed looking at performance on the individual subtests of the battery as well as reaction time and

From the Departments of Neurology and Neurosciences, Lerner Research Institute, Cleveland Clinic Foundation, OH.

Supported in part by the Movement Disorders Research Fund of the Cleveland Clinic Foundation.

Received June 7, 2000. Accepted in final form September 28, 2000.

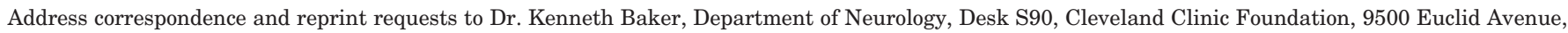
Cleveland, OH 44195; e-mail: bakerk@ccf.org 
movement velocity data derived during performance of the motor task.

Methods. Subjects. First-degree relatives were recruited either from the families of PSP patients under the care of physicians at the Cleveland Clinic Foundation or through advertisement in the national newsletter, PSP Advocate. All participating subjects were judged neurologically normal based upon interview and examination by a movement disorders specialist (E.B.M.). The examination particularly addressed symptoms and signs such as bradykinesia, tremor, flexed posture, and difficulty with walking or eye movements. Most FDR were related to patients being followed by a movement disorders specialist at the Cleveland Clinic Foundation or were relatives of subjects with PSP who had participated in previous research projects in our laboratory. Thus, the diagnosis of PSP in the affected relative had a high probability of being correct. Those FDR whose relative with PSP was not a patient at the Cleveland Clinic Foundation, and whose diagnosis had not been confirmed by autopsy elsewhere, were interviewed extensively regarding that relative. Particularly, the FDR had to describe problems with eye movements and lack of response to levodopa in the affected relative in order to be included in the study. If the FDR was not clear on the clinical history of the affected relative, he or she was asked to contact a family member with the necessary information and ask him or her to contact the laboratory. The FDR were accepted into the study only after the movement disorders expert was satisfied that the affected relative met the published diagnostic criteria for PSP. ${ }^{20}$ Although such criteria do not exclude the possibility of including patients with disorders other than PSP, they lessen the possibility.

One possible concern is the accuracy of diagnosis of PSP in the indexed cases. The main concern in this study is the possible inclusion of persons with diagnoses other than PSP, particularly PD. Previous studies have shown that first-degree relatives of patients with $\mathrm{PD}$ have a higher rate of abnormal performance on the test battery. ${ }^{1}$ Inclusion of first-degree relatives of patients with PD could have biased the results. However, the diagnostic criteria required in this study minimizes that risk. The critical issue regarding diagnostic accuracy is the rate of false positive diagnoses and not the rate of false negatives. The requirement of a documented history of lack of response to levodopa has a $96 \%$ probability of excluding patients with idiopathic PD based on the finding of Hughes et al. ${ }^{21}$ that $96 \%$ of patients with postmortem documentation of idiopathic PD had a history of responsiveness to levodopa. The additional criterion of a documented history of impairment of volitional vertical eye movements makes it highly improbable that first-degree relatives of patients affected by disease other than PSP were included in this study.

Normal control subjects were selected from a larger database of $120 \mathrm{NC}$ individuals and matched to FDR by sex and age within 5 years. The sample was selected by sorting the larger database based on subject age and selecting consecutive entries that matched both the age and sex of a FDR subject without regard to the performance of the matched subject on the test measures. Individuals in the NC group had been recruited from the staff and faculty of the University of Arizona College of Medicine, University of Kansas Medical Center, the Cleveland Clinic Foundation, or friends and non-parkinsonian members of local support groups. All NC individuals were interviewed by a movement disorders specialist or a senior research associate with extensive knowledge of parkinsonism and excluded if they had any symptoms or signs of parkinsonism such as bradykinesia, tremor, flexed posture, or difficulty with walking. NC subjects were asked directly during the interview if any of their relatives had any signs, symptoms, or a diagnosis of parkinsonism or PSP. Subjects were excluded if there was any reported family history of parkinsonism or PSP, although complete ascertainment is problematic. All individuals gave prior written informed consent and the protocol received prior approval by the institutional review boards of the Cleveland Clinic Foundation, the University of Kansas Medical Center, and the University of Arizona College of Medicine.

Other conditions that could have affected performance on the test battery were considered. Any individuals with other conditions that could affect the sense of smell (such as a history of head trauma or active rhinitis) or performance of the wrist tasks (such as arthritis) also were excluded. Recent smoking history was ascertained. In addition, any individual taking medications capable of blocking dopamine receptors or depleting dopamine stores within 3 months prior to their participation were excluded. Individuals with depression or taking medications that could cause or exacerbate depression were not specifically excluded. Previous studies indicate that the Beck Depression Inventory (BDI), one of the components in the PD Battery, does not make a significant contribution to the PD score and explains only a small percentage of the variance in the results of the PD Battery of early and mild PD patients and NC individuals. ${ }^{18}$ The BDI was retained, however, because it was used in the original development of the scoring equations.

$P D$ Battery. The PD Battery incorporates tests of motor function, olfaction, and mood, and has been described previously. ${ }^{18}$ Briefly, the motor task consists of rapid wrist flexion and extension movements made to one of two types of targets in response to an auditory "go" signal. Olfactory function was measured by the University of Pennsylvania Smell Identification Test (UPSIT, Sensonics, Inc. Haddonfield, NJ). Finally, mood state was assessed using the BDI. Results from the test battery were combined in a logistic regression analysis into an equation that yielded a score (PDscore) between 0 and 1.0 for each individual.

Results. Seven sons, 13 daughters, one brother, and two sisters (23 total) of patients with PSP, representing 11 different families, were tested. The average age was 43.5 years (range 27 to 83 ) in the FDR group and 44.3 years (range 27 to 83 ) in the group of 23 matched NC subjects. The affected relatives of the FDR were fathers in 18, mothers in 2, and brothers in 3 . None of the families reported more than one family member with known PSP; however, three of the families reported one additional relative with a diagnosis of PD. Participant characteristics are presented in the table.

None of the matched NC individuals had a PDscore $<$ 0.5 as compared to 9 of $23(39.1 \%)$ FDR who did. This difference was significant (Fisher's exact test, $p<0.001$ ). Figure 1 shows the distribution of PDscores for each group. The nine FDR who performed abnormally represented six 


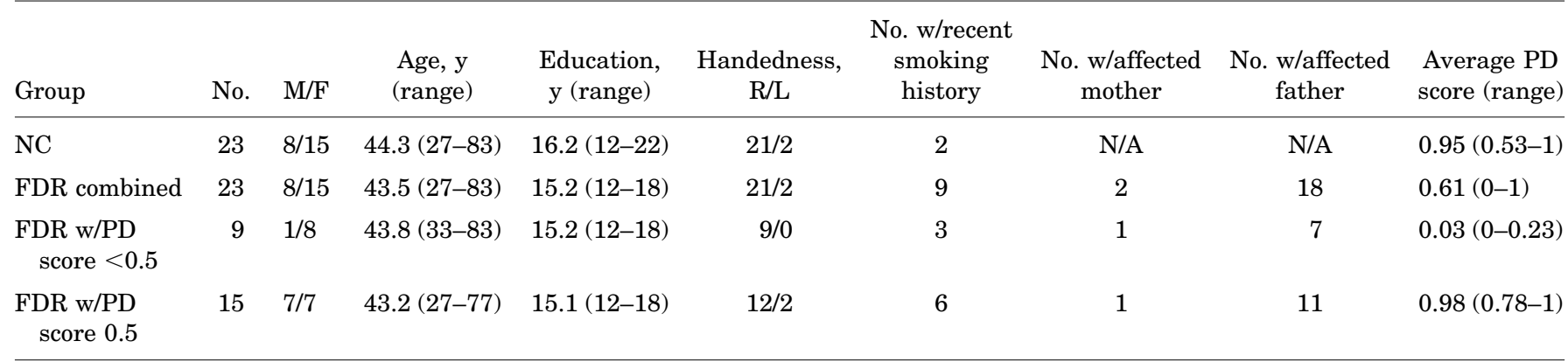

Data for the combined first-degree relative (FDR) group are shown first, then broken down by PD score.

$\mathrm{NC}=$ normal controls; N/A = not applicable.

of the 11 different families from which subjects were drawn, with three of the families having two asymptomatic study participants scoring in the abnormal range. In these three cases, however, there was at least one additional family member who scored in the normal range on the test battery. Within the 11 families sampled, three reported a single additional extended family member with a diagnosis of PD.

One $(12.5 \%)$ of eight sons and brothers tested in the abnormal range and $8(53 \%)$ of 15 sisters or daughters tested in the abnormal range. This difference did not reach significance (Fisher's exact test, $p=0.08$ ), although the power of the test was poor (0.47). Summary data are presented in the table. Among the NC individuals, there was no correlation of age with PDscore. Furthermore, there was no difference in age between those FDR whose performance on the test battery was in the abnormal range (median age $=39.5 ; 25$ th $=34.0 / 75$ th $=49.0$ ) or the normal range (median age $=39.0 ; 25$ th $=35.0 / 75$ th $=45.0$ ). Therefore, the increased rate of abnormalities in the FDR group is not due to older age.

Analysis of the results from the individual subtests revealed that the performance of the FDR was significantly different from NC individuals on all three subtests of the PD Battery. The results of the wrist task were derived from an analysis of the movement velocities in each of the four wrist movements using a regression analysis as described above. The analysis yields a single measure of performance for all four tasks. The result is a probability score (Pwrist) between 0 and 1 and reflects the probability of performing similarly to NC individuals (i.e., normal indi-

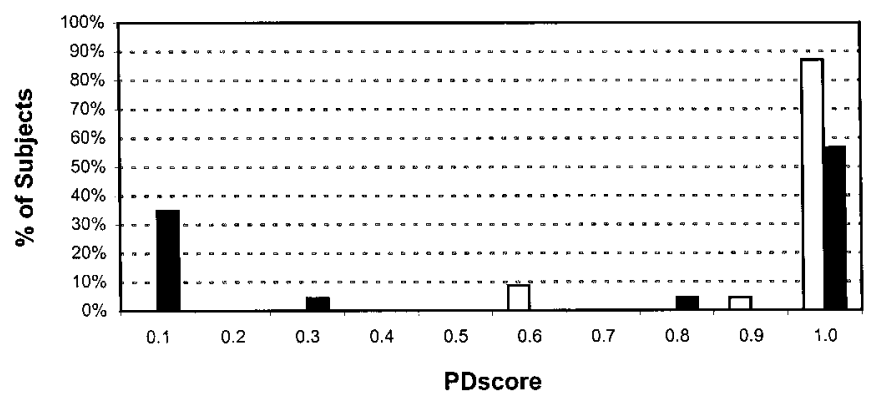

Figure 1. Graph shows the distribution of PDscores of all the first-degree relatives of patients with progressive supranuclear palsy (black bars) and normal control individuals (white bars). viduals would have a Pwrist score of 1 ). It should be noted that the analysis equations were derived from a previous study and applied prospectively to participants in this study. Consequently, any differences in the Pwrist scores between groups were not artificial because of a regression analysis.

The mean Pwrist score was $0.67( \pm 0.21)$ for the FDR group and $0.84( \pm 0.17)$ for the NC group. The difference was significant (unpaired $t$-test, $p<0.01$ ). The mean ageand sex-corrected percentile score for the UPSIT for the FDR group was $48 \%( \pm 28.4)$ whereas the mean score for the NC individuals was $68 \%( \pm 26.5)$ (unpaired $t$-test, $p=$ 0.017). The median BDI for the FDR group was $4.5(25 \%$ $2.0 / 75 \% 7.1$ ) whereas the median score for the NC individuals was $2.0(25 \%$ 0.0/75\% 4.1) (Mann-Whitney rank sum test, $p=0.015$ ).

Although not a part of the regression equations used to derive the PDscore, the motor task further provides information on subject reaction time and movement velocity for each of the four task conditions. An analysis of variance of repeated measures design revealed no significant difference in reaction time for each of the four task conditions. As such, the reaction time data were averaged across the four conditions for each subject. Figure 2 shows the distribution of reaction times for the two groups. The median reaction time was 38.0 (first quartile 30.9/third quartile 53.0) for the FDR group and 30.5 (first quartile 24.6/third quartile 33.4) for the NC group. This difference was significant (Mann-Whitney rank sum test, $p<0.001$ ).

Figure 3 shows the distribution of movement velocities for the two groups. The distribution of the movement ve-

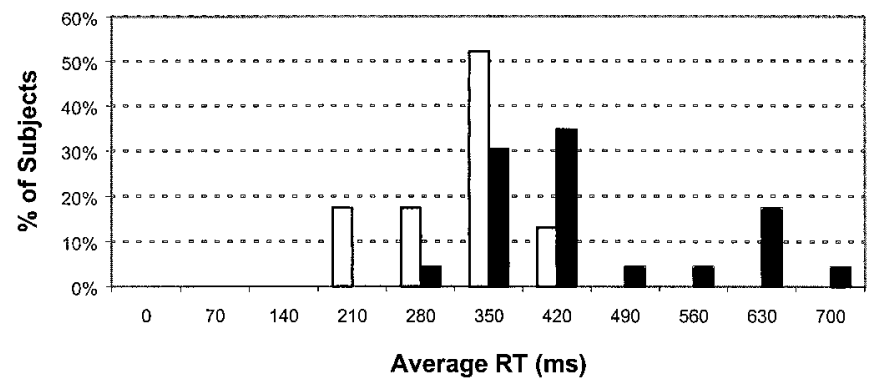

Figure 2. Graph shows the distribution of reaction times (RT) in all the first-degree relatives of patients with progressive supranuclear palsy (black bars) and normal control individuals (white bars). 


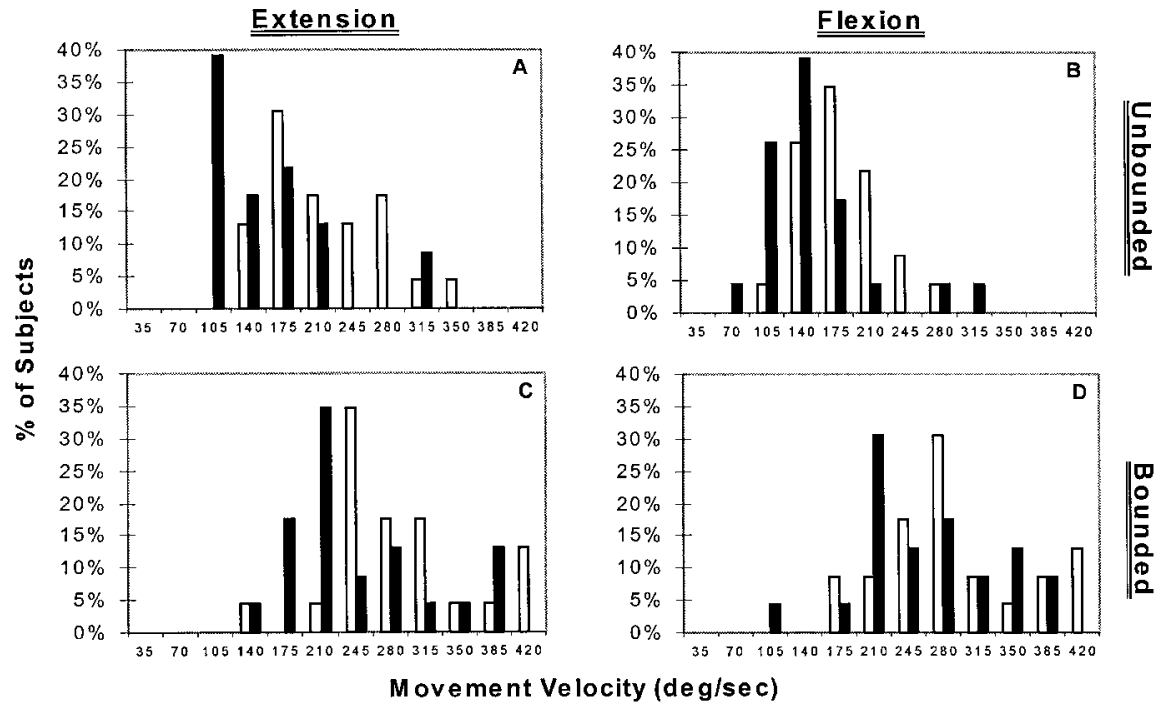

Figure 3. Graphs showing the distribution of movement velocities for the firstdegree relatives of patients with progressive supranuclear palsy (FDR) and normal control (NC) groups for each of the four movement patterns of the wrist task. During the task, an auditory "go" signal was used to cue the subject to produce either flexion ( $A$ and $C$ ) or extension $(B$ and $D)$ of the wrist from the start point to the target position. The target position was either unbounded (A and B), requiring that the subject stop the movement, or bounded (C and D) by a mechanical stop, beyond which the movement could not be continued. Black bars $=F D R$; white bars $=$ NC. locities was skewed. Consequently, movement velocities were logarithmically transformed, resulting in a normal distribution to permit parametric statistical analysis to examine the difference in movement velocities. An analysis of variance of repeated measures design resulted in a significant group $(p=0.002)$ and task $(p<0.001)$ difference as well as a group by task interaction $(p=0.03)$ in the mean of the logarithmically transformed movement velocities. The FDR subject group was consistently slower than the NC group on each of the four movement patterns. Using unpaired $t$-tests, this difference was found to be significant for the extension unbounded ( $p<0.001$ ), extension bounded ( $p=0.025)$, and flexion unbounded ( $p=$ 0.012 ) movement patterns.

Discussion. The FDR of patients with PSP had a significantly higher prevalence of abnormalities on the PD Battery than did NC subjects without a family history of movement disorders. Consistent with this was the finding that the performance of the two groups was significantly different across all three subtests of the battery. It is impossible to know at this point which, if any, of the FDR might go on to develop PSP at some point in the future. The increasing number of familial cases being reported in the literature suggests that there may be some increase in risk, but just how much is not clear. There was no family history of PSP beyond the single index case for each of the FDR participating in this study. In any case, what is clear is that many of the FDR testing in the abnormal range in the current study are unlikely to go on to develop PSP, suggesting that the PD Battery may be detecting an asymptomatic carrier state or subclinical form of the disease.

Investigations into the factors responsible for PSP, whether focused on genetics or environmental toxins, are complicated by the late onset and rarity of the disease as well as the limited reliability of historical information from families. Moreover, similar to what has been observed in PD, there may be a familial form of PSP that differs genetically from the more typical and seemingly sporadic form of the dis- ease. Indeed, the pattern of inheritance suggested by reports in the literature has been mixed, with both recessive $^{5,22}$ and dominant ${ }^{3,4,6}$ patterns observed. Other familial case reports are not sufficiently complete to allow a confident determination to be made. ${ }^{7}$ However, a recent investigation examining the frequency of tau polymorphisms in PSP patients with no family history of the disease showed evidence of linkage disequilibrium between PSP and the tau marker using a recessive as opposed to a dominant model of inheritance. ${ }^{15}$ Although certainly complicated by the factors mentioned previously, this study provides some evidence that the more sporadic variety of PSP may be recessively inherited with variable penetrance.

If we assume an autosomal recessive mode of transmission, then $25 \%$ or approximately 6 of the 23 FDR tested in the current study would be expected to carry the putative gene or be at risk. Alternatively, an autosomal dominant pattern suggests that $50 \%$ or approximately 12 of the 23 subjects should be at risk. The actual prevalence of abnormalities in the FDR tested was $39 \%$ or 9 of 23 , a figure that falls about midway between the different models. The absence of false positives in the matched NC group, although worthy of note, does not bear a significant impact on this finding. Within the larger database of $120 \mathrm{NC}$ individuals from which the matched subjects were selected, the total false positive rate is $9 \%$. Although there is no correlation between age and PD score in that group $(r=-0.13, p=0.144, \mathrm{n}=120)$, the false positive rate for individuals under 52 years of age is only $3.1 \%$. Given that 20 of the 23 FDR of PSP patients were 51 years of age or younger, it is not surprising that the NC sample should be without false positives. Even if we were to allow for a $9 \%$ false positive rate in $\mathrm{NC}$ individuals, this would predict that only 1 of the 11 FDR without the gene would have a false positive abnormality using the dominant model or 2 of 17 using the recessive model. Thus, the PD Battery would have accurately identi- 
fied $66 \%$ (8 of 12) under the autosomal dominant model and $42 \%$ (7 of 17) using the autosomal recessive model-much higher than the rather conservative $9 \%$ false positive rate in the NC sample. Finally, a sex-linked inheritance pattern does not seem likely; however, the power of the performed test was insufficient to completely rule out such a pattern in this small sample.

The observed difference in olfactory function between the two groups is of interest given the lack of olfactory findings in patients with PSP. Reports in the literature have shown that the odor identification ability of patients with PSP is comparable to normal control subjects and significantly better than patients with idiopathic PD. ${ }^{23,24}$ However, in reviewing both reports it is clear that there is a marked trend toward reduced olfactory function in the patients with PSP. Neither set of authors reported the results of subsequent power analysis, leaving open the possibility of a type II error in their results. That is to say, the possibility exists that the null hypothesis, which in this case would state that there is no difference between the groups, may have been falsely accepted. The higher rate of smoking in the FDR group is of some concern given the potential impact of smoking on the sense of smell. However, the PDscore reflects performance on all three subtests and abnormal performance on any single subtest of the battery will not result in an abnormal score. The observed difference on the BDI is not surprising, given previous reports of psychiatric symptoms, including depression-like symptoms, in patients with PSP. ${ }^{25-26}$

Reaction time was observed to be significantly longer in the FDR group as compared to the NC group across all tasks. If we assume the possibility of a subclinical disease state or an asymptomatic carrier state in PSP, there is both theoretical and empirical evidence that coincides with this finding. Previous studies have suggested that motor initiation utilizes physiologic mechanisms separate from those underlying motor execution..$^{27,28}$ These studies have suggested that the anterior striatum, consisting of the head of the caudate nucleus and the anterior putamen, may be more involved in motor initiation, whereas the posterior striatum is more involved in motor execution. PET and SPECT have shown preservation of dopamine in the anterior striatum relative to posterior striatum of PD compared to PSP patients. ${ }^{29,30}$ Several groups have demonstrated that reaction time is delayed in patients with $\mathrm{PSP},{ }^{31-34}$ even in those with relatively mild disease. ${ }^{33}$ All of this suggests that reaction time may be of some value in further improving the identification of PSP as well as the asymptomatic carrier state or subclinical form of the disease.

There was an observed trend in the current study for extension movements to be more affected than flexion movements in the FDR group. This is consistent with observations in experimental animal studies. Denny-Brown ${ }^{35}$ showed that nonhuman primates be- came immobile in a flexed posture following large lesions of the globus pallidus. Similarly, injections of muscimol, a gamma-aminobutyric acid (GABA) agonist that inactivates the globus pallidus, have been shown to produce a greater slowing of extension movements compared to flexion movements on a wrist flexion and extension task similar to that used in the current study ${ }^{36}$ Finally, recordings of neuronal activity changes made in nonhuman primates and correlated with wrist flexion and extension movements before and following induction of parkinsonism using n-methyl-4phenyl-1,2,3,6-tetrahyrdopyridine (MPTP) showed that greater changes in neuronal activity following MPTP were associated with the wrist extension task than with the flexion task. ${ }^{37}$

One possible explanation for the greater impairment of extension movements may be that there is a greater representation or dedication of neurons to flexion motor control in the basal ganglia. This greater representation could explain the predominance of flexion after stimulation and may convey increased resistance to degradation of performance of flexion movements. Thus, flexion is relatively well preserved, resulting in a flexor bias such as flexed posture. Also, there would be greater impairment of extension movements with disease.

The results of the current study are of considerable interest regardless of whether the pathogenesis of PSP involves genetic or environmental factors. In either case, the PD Battery, either in its present form or with the addition of reaction time data, could help advance research into the cause of PSP. If the cause is genetic, then the PD Battery may be able to detect the asymptomatic carrier state or risk. Comparing the genetic makeup of the FDR scoring in the abnormal range with that of the unaffected parents or siblings who score in the normal range could lead to the identification of a shared genetic makeup that could cause or facilitate PSP. Likewise, if the cause is environmental, the PD Battery may be able to detect preclinical or subclinical involvement. As such, comparing the environmental exposure of FDR who score in the abnormal range with those who do not may help to identify potential causative agents. Further, the presumably earlier detection would be closer to the time of exposure, thereby facilitating the discovery of causative environmental factors.

\section{Acknowledgment}

The authors thank John Gale for his assistance.

\section{References}

1. Montgomery EB Jr, Baker KB, Lyons K, Koller WC. Abnormal performance on the PD test battery by asymptomatic firstdegree relatives. Neurology 1999;52:757-762.

2. Lantos PL. The neuropathology of progressive supranuclear palsy. J Neural Transm 1994;42(suppl):137-152.

3. de Yébenes JG, Sarasa JL, Daniel SE, Lees AJ. Familial progressive supranuclear palsy: description of a pedigree and review of the literature. Brain 1995;118:1095-1103.

4. Gazeley S, Maguire J. Familial progressive supranuclear palsy. Clin Neuropathol 1996;15:215-220. 
5. Ohara S, Kondo K, Morita H, et al. Progressive supranuclear palsy-like syndrome in two siblings of a consanguineous marriage. Neurology 1992;42:1009-1014.

6. Rojo A, Pernaute RS, Fontán A, et al. Clinical genetics of familial progressive supranuclear palsy. Brain 1999;122:1233-1245.

7. Tetrud JW, Golbe LI, Forno LS, Farmer PM. Autopsy-proven progressive supranuclear palsy in two siblings. Neurology 1996;46:931-934.

8. Baker M, Litvan I, Houlden H, et al. Association of an extended haplotype in the tau gene with progressive supranuclear palsy. Hum Mol Genet 1999;8:711-715.

9. Chambers CB, Lee JM, Troncoso JC, et al. Overexpression of four-repeat tau mRNA isoforms in progressive supranuclear palsy but not in Alzheimer's disease. Ann Neurol 1999;46: 325-332.

10. Conrad C, Andreadis A, Trojanowski JQ, et al. Genetic evidence for the involvement of tau in progressive supranuclear palsy. Ann Neurol 1997;41:277-281.

11. Delisle MB, Murrell JR, Richardson R, et al. A mutation at codon $279(\mathrm{~N} 279 \mathrm{~K})$ in exon 10 of the tau gene causes tauopathy with dementia and supranuclear palsy. Acta Neuropathol 1999;98:62-77.

12. Ezquerra M, Pastor P, Valldeoriola F, et al. Identification of a novel polymorphism in the promoter region of the tau gene highly associated to progressive supranuclear palsy in humans. Neurosci Lett 1999;275:183-186.

13. Higgins JJ, Adler RL, Loveless JM. Mutational analysis of the tau gene in progressive supranuclear palsy. Neurology 1999; 53:1421-1424.

14. Higgins JJ, Litvan I, Nee LE, Loveless JM. A lack of the R406W tau mutation in progressive supranuclear palsy and corticobasal degeneration. Neurology 1999;52:404-406.

15. Higgins JJ, Litvan I, Pho LT, et al. Progressive supranuclear palsy is a recessive disorder in linkage disequilibrium with the tau and not the alpha-synuclein gene. Neurology 1998;50: $270-273$.

16. Lazzarini AM, Golbe LI, Dickson DW, et al. Tau intronic polymorphism in Parkinson's disease and progressive supranuclear palsy. Neurology 1997;48(suppl):A427. Abstract.

17. Marconi R, Meco G, Nicholl DJ, et al. Direct genetic evidence for involvement of tau in progressive supranuclear palsy. European Study Group on Atypical Parkinsonism Consortium. Neurology 1998;51:982-985.

18. Mongomery EB Jr, Koller WC, LaMantia TJ, et al. Early detection of probable idiopathic Parkinson's disease: I. Development of a diagnostic test battery. Mov Disord 2000;15:467473.

19. Montgomery EB Jr, Lyons K, Koller WC. Early detection of probable idiopathic Parkinson's disease: II. A prospective application of a diagnostic test battery. Mov Disord 2000;15: $474-478$.

20. Litvan I, Agid Y, Calne D, et al. Clinical research criteria for the diagnosis of progressive supranuclear palsy (SteeleRichardson-Olszewski syndrome): report of the NINDS-SPSP International Workshop. Neurology 1996;47:1-9.
21. Hughes AJ, Daniel SE, Kilford L, Lees AJ. Accuracy of clinical diagnosis of idiopathic Parkinson's disease: a clinico-pathological study of 100 cases. J Neurol Neurosurg Psychiatry 1992;55:181184.

22. Brown J, Lantos P, Stratton M, Roques P, Rossor M. Familial progressive supranuclear palsy. J Neurol Neurosurg Psychiatry 1993;56:473-476.

23. Doty RL, Golbe LI, McKeown DA, et al. Olfactory testing differentiates between progressive supranuclear palsy and idiopathic Parkinson's disease. Neurology 1993;43:962-965.

24. Wenning GK, Shephard B, Hawkes C, et al. Olfactory function in atypical parkinsonian syndromes. Acta Neurol Scand 1995; 91:247-250.

25. Esmonde T, Giles E, Gibson M, Hodges JR. Neuropsychological performance, disease severity, and depression in progressive supranuclear palsy. J Neurol 1996;243:638-643.

26. Menza MA, Cocchiola J, Golbe LI. Psychiatric symptoms in progressive supranuclear palsy. Psychosomatics 1995;36:550 554 .

27. Montgomery Jr. EB, Gorman D, Nuessen J. Motor initiation versus execution in normal and Parkinson's disease subjects. Neurology 1991;41:1469-1475.

28. Montgomery EB, Nuessen J, Gorman D. Reaction time and movement velocity abnormalities in Parkinson's disease under different task conditions. Neurology 1991;41:1476-1481.

29. Messa C, Volonté MA, Fazio F, et al. Differential distribution of striatal $\left[{ }^{123} \mathrm{I}\right] \beta$-CIT in Parkinson's disease and progressive supranuclear policy, evaluated with single-photon emission tomography. Eur J Nucl Med 1998;25:1270-1276.

30. Ilgin N, Zubieta J, Reich SG, et al. PET imaging of the dopamine transporter in progressive supranuclear palsy and Parkinson's disease. Neurology 1999;52:1221-1226.

31. Bisdorff AR, Bronstein AM, Wolsley C, Gresty MA, Davies A, Young A. EMG responses to free fall in elderly subjects and akinetic rigid patients. J Neurol Neurosurg Psychiatry 1999; 66:447-455.

32. Dubois B, Pillon B, Legault F, Agid Y, Lhermitte F. Slowing of cognitive processing in progressive supranuclear palsy. A comparison with Parkinson's disease. Arch Neurol 1988;45:11941199.

33. Johnson R, Litvan I, Grafman J. Progressive supranuclear palsy: altered sensory processing leads to degraded cognition. Neurology 1991;41:1257-1262.

34. Valldeoriola F, Valls-Sole J, Tolosa E, Ventura PJ, Nobbe FA, Marti MJ. Effects of a startling acoustic stimulus on reaction time in different parkinsonian syndromes. Neurology 1998;51: 1315-1320.41.

35. Denny-Brown D. The basal ganglia, and their relation to disorders of movement. London: Oxford University Press, 1962.

36. Mink JW, Thach WT. Basal ganglia motor control. III. Pallidal ablation: normal reaction time, muscle cocontraction, and slow movement. J Neurophysiol 1991;65:330-351.

37. Montgomery EB Jr. Abnormal dynamic modulation of putamen neuronal activity following MPTP. Soc Neurosci Abstracts 1993;19:1051. Abstract. 


\section{Neurology}

\section{Performance on the PD test battery by relatives of patients with progressive supranuclear palsy}

Kenneth B. Baker and Erwin B. Montgomery, Jr.

Neurology 2001;56;25-30

DOI 10.1212/WNL.56.1.25

\section{This information is current as of January 9, 2001}

\section{Updated Information \& Services}

Supplementary Material

\section{References}

\section{Citations}

Subspecialty Collections

Permissions \& Licensing

Reprints including high resolution figures, can be found at: http://n.neurology.org/content/56/1/25.full

Supplementary material can be found at: http://n.neurology.org/content/suppl/2000/12/11/56.1.25.DC1 http://n.neurology.org/content/suppl/2000/12/11/56.1.25.DC2

This article cites 36 articles, 17 of which you can access for free at: http://n.neurology.org/content/56/1/25.full\#ref-list-1

This article has been cited by 1 HighWire-hosted articles: http://n.neurology.org/content/56/1/25.full\#\#otherarticles

This article, along with others on similar topics, appears in the following collection(s):

\section{All Genetics}

http://n.neurology.org/cgi/collection/all_genetics

All Movement Disorders

http://n.neurology.org/cgi/collection/all_movement_disorders

Parkinson's disease/Parkinsonism

http://n.neurology.org/cgi/collection/parkinsons_disease_parkinsonism Progressive supranuclear palsy

http://n.neurology.org/cgi/collection/progressive_supranuclear_palsy

Information about reproducing this article in parts (figures,tables) or in its entirety can be found online at:

http://www.neurology.org/about/about_the_journal\#permissions

Information about ordering reprints can be found online:

http://n.neurology.org/subscribers/advertise

Neurology ${ }^{\circledR}$ is the official journal of the American Academy of Neurology. Published continuously since 1951, it is now a weekly with 48 issues per year. Copyright . All rights reserved. Print ISSN: 0028-3878. Online ISSN: 1526-632X.

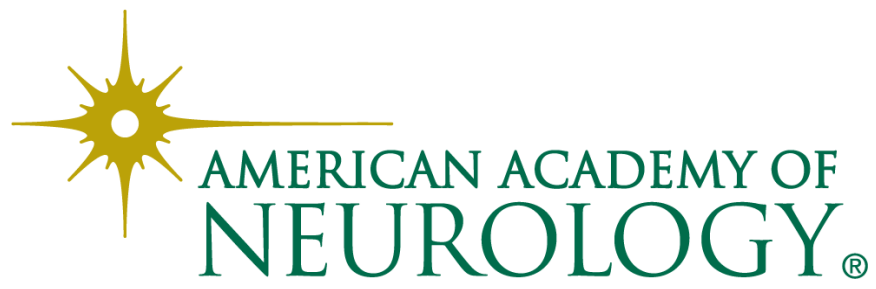

